FETAL AND NEONATAL EDITION

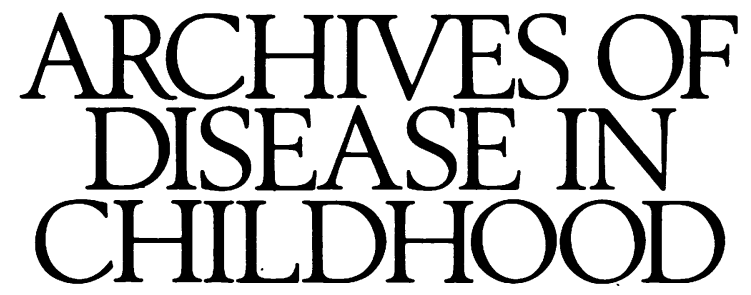

The fournal of the British Paediatric Association

\title{
Fetal activity, behaviour, and neurology: fact or fiction?
}

Behaviour is the interaction between environment and activity. In the newborn infant, behaviour has been categorised by Prechtl in a series of behavioural 'states'. Prechtl has defined them as 'distinct and mutually exclusive conditions, each having its specific properties and reflecting a particular mode of nervous function'. ${ }^{1}$ The newborn cycles between state epochs which are both stable and recurring. Neonatal behavioural state is determined by both direct observation of movement and activity patterns (table) and by polygraphic recordings of the electroencephalogram and other physiological variables.

It has been shown that the premature infant shows the consistent behavioural patterns defined by Prechtl from 36 weeks' postmenstrual age onwards, ${ }^{23}$ and it is reasonable to believe that the fetus of 36 weeks also shows these patterns of activity developing towards term. Nijhuis et al were the first to describe fetal 'behaviour' by using two real time ultrasound machines to assess simultaneously fetal body, face, and eye movements as well as fetal breathing patterns. ${ }^{4}$ They also recorded fetal heart rate variability and related changes in this variable to movement. It has subsequently been suggested that in order to record at least one complete activity cycle, the minimum observation period should be 1-2 hours. ${ }^{56}$ Using these methods of assessing fetal activity, four fetal 'behavic-ural' states have been described ${ }^{4}$ and are shown in the table. These have been adapted from Prechtl's neonatal behavioural states. Nijhuis states that the fetus cannot be considered to be awake in the uterus, ${ }^{7}$ but many describe fetal states as being either 'asleep' or 'awake'.

The description of fetal state, and by implication

Neonatal and fetal behavioural states

Neonatal behavioural states ${ }^{11}$

1 Eyes closed, regular respiration, no movements

2 Eyes closed, irregular respiration, no gross movements

3 Eyes open, no gross movements

4 Eyes open, no gross movements

4 Eyes open, continual gross movements,

Fetal behavioural states 4

No body movements, except startles, and no eye movement

Frequent and periodic gross body movements with

continuous eye movement

Absent gross body movements with continuous eye

movement

4 Vigorous continual body movements with continuous eye

movement behaviour, is compelling to many obstetricians and others, but it is clear that fetal state is not directly comparable with neonatal state in two major ways. Firstly, electrophysiological activity from the cerebral cortex and muscles that are considered fundamental to the determination of state in the newborn are not accessible in the fetus. Secondly, it is not possible to recognise fetal 'sleep' in the way that sleep can be recognised in the neonate. As indicated in the definition of behaviour, activity is modulated at least to some extent by environmental factors. Although the fetus of 40 weeks and the newborn full term infant have the same patterns of neurological activity, the environmental stimuli vary considerably to the point that the two are not comparable.

In my view, these fundamental problems in defining fetal state throw considerable doubt on the scientific validity of techniques that attempt to assess fetal behaviour. This is not to say that fetal activity cannot be closely assessed, this is clearly the case, but simply that the inference that these patterns of activity reflect fetal 'behaviour' must be questioned.

It follows from this that if fetal behaviour cannot be defined in the mature fetus, where stable behavioural states can be observed in neonates of the same gestational age, then the more immature the fetus, the more unlikely it is that epochs of activity represent cortically modulated 'behaviour'. The observed movements probably represent patterns of reflex activity.

\section{The assessment of fetal neurology}

Neurological assessment involves observation, interaction with the patient to assess orientation and conscious state, assessment of tone and reflexes, and integration of these functions in activities. Assessment of the fetus allows observation of a limited number of functions (body and eye movements, fetal heart rate patterns, breathing, etc) which reflect components of activity but these individual features cannot be integrated into an overall assessment of 'fetal neurology'.

Recently attempts have been made to assess fetal behaviour with the aim of detecting the neurologically abnormal fetus. ${ }^{8}$ In this issue Horimoto and colleagues report 10 immature fetuses who were thought to show abnormal behavioural features or who were found to have 
overt brain abnormalities on ultrasound scans. ${ }^{9}$ They asked whether abnormal in utero behavioural patterns could be used as a test of neurological abnormality. Two of the 10 infants were found to be subsequently normal. This very interesting paper raises the question of whether fetal activity can be assessed and whether this can be related to neurological abnormality.

I believe that the term 'fetal behaviour' is misleading and has no scientific validity. Nevertheless, it is valid to ask whether assessment of fetal activity can recognise a group of fetuses who later show neurological abnormality. This will be a very difficult question to answer. We know that the infant can show abnormal neurological features which recover and the child is eventually functionally normal. Thus clinical assessment may not predict outcome and abnormal fetal activity will therefore be extremely difficult to measure against a gold standard. We require longitudinal studies of groups of fetuses who have careful assessment of activity and relate these abnormalities to eventual outcome at 1 to 2 years of age.

The assessment of fetal activity is a fascinating area for future research, but we must not overinterpret the preliminary findings. Activity is not the same as behaviour and fetal neurology is not the assessment of activity alone. Moore and Hanson in reviewing this theme have concluded that the 'absence of evidence is not in itself evidence of absence'. ${ }^{10}$

Academic Unit of Paediatrics and Child Health, MALCOLM I LEVENE

$D$ Floor, Clarendon Wing,

General Infirmary at Leeds,

Belmont Grove, Leeds LS2 9NS

1 Prechtl HFR. The behavioural states of the newborn infant (a review). Brain Res 1974; 76: 185-212.

2 Parmalee AH, Stern E. Development of states in infants. In: Clemente C, Purpura D, Meyer F, eds. Sleep and the maturing nervous system. New York: Academic Press, 1972: 199-228.

3 Prechtl HFR, Fargel JW, Weinmann HM, Bakker HH. Posture, motility and respiration in low-risk preterm infants. Dev Med Child Neurol 1979; 21: 3-27.

4 Nijhuis JG, Prechtl HFR, Martin CB, Bots RSGM. Are there behavioural states in the human fetus? Early Hum Dev 1982; 6: 177-95

5 Timor-Tritsch IE, Dierker LJ, Hertz RH, Deagan C, Rosen MG. Studies of antepartum behavioural states in the human fetus at term. Am $\mathcal{F}$ Obstet Gynecol 1978; 132: 524-8.

6 Visser GHA, Dawes GS, Redman CWG. Numerical analysis of the normal human fetal heart rate. Br $\mathcal{F}$ Obstet Gynaecol 1981; 87: 792-802.

7 Nijhuis JG. The third trimester. In: Nijhuis JG, ed. Fetal behaviour: developmental and perinatal aspects. Oxford: Oxford Medical Publications, 1992: $26-40$.

8 Pillai M, Garrett C, James D. Bizarre fetal behaviour associated with lethal congenital anomalies: a case report. Eur f Obstet Gynecol Reprod Biol 1991; 39: $215-8$

9 Horimoto N, Koyanagi T, Maeda H, et al. Can brain impairment be detected by in utero behavioural patterns? Arch Dis Child 1993; 69: 3-8.

10 Moore PJ, Hanson MA. Animal investigations. In: Nijhuis JG, ed. Fetal behaviour: developmental and perinatal aspects. Oxford: Oxford Medical Publications, 1992: 100-11.

11 Prechtl HFR, Beintema DJ. The neurological examination of the full-term newborn infant. Clinics in developmental medicine. No 12 . London: Heinemann, 1964. 